\title{
Rapid cycling is equivalently prevalent in bipolar I and bipolar II disorder, and is associated with female gender and greater severity of illness
}

Schneck CD, Miklowitz DJ, Calabrese JR, et al. Phenomenology of rapid cycling bipolar disorder: Data from the first 500 participants in the Systematic Treatment Enhancement Program. Am J Psychiatry 2004;161:1902-8.

How prevalent is rapid cycling in people with bipolar disorder and do demographic and clinical variables differ between rapid cyclers and non-rapid cyclers?

\section{METHODS}

$\square$

Design: Cross-sectional study

(19)

Setting: USA; time frame not stated.

으으 Population: 456 individuals aged at least 15 years old, with bipolar I or bipolar II disorder (DSM-IV) enrolled in the Systematic Treatment Enhancement Program for Bipolar Disorder. 75.7\% participants had bipolar I disorder and $24.3 \%$ had bipolar II disorder; $40.6 \%$ were men.

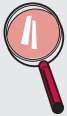

Assessment: The Affective Disorder Evaluation and the MiniInternational Neuropsychiatric Interview were used to diagnose bipolar disorders. In addition, the Young Mania Rating Scale (YMRS), the Montgomery-Åsberg Depression Rating Scale (MADRS), and the Global Assessment of Functioning Scale (GAFS) were used to assess disorder severity, presence of psychosis, and number of affective episodes. Demographic and symptomatic characteristics were compared using the nonparametric Wilcoxon test or the $\chi^{2}$ test. The generalised linear model was used to assess the interaction of rapid cycling and lifetime diagnosis with continuous variables, and logistic regression used to examine the interaction with discrete outcome variables.

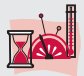

Outcomes: Prevalence of rapid cycling bipolar disorder (four or more syndromal depressive, manic, or hypomanic episodes in the previous 12 months); illness severity: MADRS-higher score indicates greater severity, YMRS - higher score indicates greater severity, GAFS - higher score indicates better function

\section{MAIN RESULTS}

Twenty per cent of bipolar participants (91/456) had rapid cycling bipolar disorder. There was no significant difference between people with bipolar I or bipolar II disorder in the prevalence of rapid cycling $(20 \%(69 / 345)$ in bipolar I disorder $v 19.8 \%(22 / 111)$ in bipolar II disorder; $\mathrm{p}=0.97)$. Women were more likely to be rapid cyclers than men $(23 \%(61 / 271)$ of women $v 16 \%(30 / 185)$ of men; $\mathrm{p}<0.04)$. Rapid cyclers had an earlier onset of illness than non-rapid cyclers (mean age at first depression: 16.7 years rapid cyclers $v 20.0$ years non-rapid cyclers, $\mathrm{p}=0.0 \mathrm{l}$; mean age at first hypomania/mania: 18.8 years rapid cyclers $v 22.1$ years non-rapid cyclers, $\mathrm{p}<0.02$ ). Rapid cyclers had more severe depression and mania, and had lower global functioning (rapid cyclers $v$ non-rapid cyclers: mean MADRS score:

For correspondence: $\mathrm{Dr} \mathrm{C} D \mathrm{D}$ Schneck, Department of Psychiatry, University of Colorado Health Sciences Center, 4455 East 12th Ave., Box A011-15, Denver, CO 80220, USA; christopher.schneck@uchsc.edu

Sources of funding: National Institute of Mental Health, USA.
$19.3 v 12.8, \mathrm{p}=0.0001$; mean YMRS score: $8.6 v 4.8, \mathrm{p}<0.0001$; mean GAFS score: $58.3 \vee 63.2$ for non-rapid cyclers, $\mathrm{p}=0.004)$.

\section{CONCLUSIONS}

Rapid cycling is equally prevalent in bipolar I and bipolar II disorder. Rapid cyclers are more likely to be women, and to have a greater severity of illness than those without rapid cycling.

\section{Commentary}

The birth of the concept of "rapid cycling" bipolar disorder was marked over 30 years ago by the publication of Dunner and Fieve's report of lithium resistance in people with a high frequency of pathological mood changes. ${ }^{1}$ During the subsequent three decades the phenomenon of rapid cycling has been widely accepted and its significance as a course modifier, particularly with respect to pharmacological response, has attracted considerable research interest. ${ }^{2}$ However, disparate definitions of rapid cycling within the literature, and other methodological limitations of published studies, have confounded attempts to define precisely the phenomenological presentations, clinical descriptions, and comorbid conditions associated with rapid cycling bipolar disorder. Given the problem of poor response to a variety of pharmacological compounds, and reports of poorer global functioning in people with rapid cycling bipolar disorder, there is a clear and urgent need for methodologically robust studies designed to address these areas of uncertainty.

The paper by Schneck et al is one of the larger studies examining demographic, historical, and symptomatic features of bipolar patients with and without rapid cycling. The individuals enrolled in the Systematic Treatment Enhancement Program for Bipolar Disorder are well characterised, but are recruited from academic and tertiary care centres in the USA, thus limiting the applicability of the findings to community samples. Notwithstanding, this study confirms several previous findings, namely: rapid cycling affects around $20 \%$ of patients with bipolar disorder, is more common in females, and is associated with poorer global functioning. However, several findings are at variance with other published reports: rapid cyclers were younger at study entry, and bipolar disorder emerged at a younger age. Also, there was no difference in the prevalence of rapid cycling between those with bipolar I and bipolar II disorder, a finding which is in agreement with some, but not all, previously published studies. Unfortunately, this study was not able to examine the relationship between rapid cycling and antidepressant use - an area of some controversy. This study highlights the need for vigilance in detecting the rapid cycling subtype of bipolar disorder, and for well designed studies of pharmacological and psychological therapies in this specific population.

Dr Paul Mackin, MB BS, PhD, MRCPsych School of Neurology Neurobiology and Psychiatry, University of Newcastle upon Tyne, UK

1 Dunner DL, Fieve RR. Clinical factors in lithium carbonate prophylaxis failure. Arch Gen Psychiatry 1974;30:229-33.

2 Mackin P, Young AH. Rapid cycling bipolar disorder: historical overview and focus on emerging treatments. Bipolar Disord 2004;6:523-9. 